\title{
Some Implications of Trade Liberalization for Choice of Trading Partners
}

\author{
Michael J. Ryan \\ University of Hull
}

\begin{abstract}
It is well known that nations potentially generate mutual gains from exchange following reductions in tariffs on commodities potentially traded between them. It might appear to follow that successive enlargements of a market by reductions in tariffs for potentially entering members would lead to Pareto improvements both for existing and potential members. The main purpose of this paper is to present variable trading bloc extensions of results analogous to the well known results by Kemp and Wan to show how, while true for particular cases, the statement in the previous sentence is not true in general. Other contributions of the paper are some implications for processes of EC enlargement as well as extensions to mobile factor cases.
\end{abstract}

- JEL Classifications: F1, F2

- Key words: Trade Liberalization, EC Enlargement

\section{Introduction}

This paper has two purposes. The first is to present general results on customs unions and free trade areas. These results can be seen as variants of the work of Kemp and Wan (1986) on customs unions and of subsequent refinements of that work by Kemp (2000) with reference to the Pareto dominance of free trade areas over customs unions and by Kemp and Shimomura (2001) with reference to

\footnotetext{
*Corresponding address: Department of Economics, Hull University Business School, University of Hull, Hull HU6 7RX, U.K. Tel: +44 (0)1482 465707, Fax: +44 (0)1482 466216, E-mail: M.J.Ryan@hull.ac.uk (2005-Center for International Economics, Sejong Institution, All Rights Reserved.
} 
nations initially excluded from a customs union. Not only are analogous results obtained here but the method used to obtain them makes it easy to show that those results also extend to cases in which factors are mobile between nations. That type of extension has not been considered by authors using the Kemp-Wan framework but is one which may be significant in practice - for example via accords concerning capital and labour mobility between certain nations in the EC. The second purpose of the paper is to show that, whether or not a Pareto dominance criterion is the one to be adopted, then in general it will matter whether or not countries join a customs union in blocs and in general it will matter, too, in what order those blocs do, or do not join the union.

The paper is organized as follows: After an informal motivation of subsequent results in Section 2, the key result is established in Section 3. In Section 4 three variants of that result are considered with reference to free trade areas, with reference to a complementary trading bloc and with reference to more comprehensive cases in which labour and capital may be mobile. Up to that point in the paper the focus will be on specifications generating Pareto improvements for all nations. In Section 5 attention will turn to processes of augmentation of trading blocs and cases in which such augmentations may or may not be Pareto improving for nations in a complementary bloc - whose membership is consequentially reduced. With that context Section 6 will draw on ideas from game theory to show why in general it will matter in what numbers and in what sequence nations do or do not join a customs union.

\section{Gains from exchange: A preliminary motivation}

The content of the remainder of the paper can be motivated informally as follows. Consider three individuals each located on a different island (of which they are the only inhabitant). Each islander is endowed with quantities of labour and of a distinct island-specific type of capital. Consider an initial situation in which there is no possibility of exchange between them - an initial state analogous to one of prohibitively large tariffs. Next consider a situation where one of those tariffs is reduced. Then there is an opportunity for potentially mutually preferable gifts to the island associated with that reduction and/or, if that tariff reduction is reciprocated by others, there may be opportunities for mutually preferred exchanges. Assuming for simplicity that all three individuals are selfish and will only exchange by means of trade, then one outcome may be trade between two 
islands and none with the third. In such a case if the third islander subsequently reduces one or more of its tariffs, potentially it will gain relative to its nontrading position by entering into trade with one or other of the two already trading islands.

At first sight it might seem that successive relaxations of tariffs would necessarily lead to at least weakly Pareto improving states for the three islanders. Indeed that would be the case for members of an existing trading bloc (here two islanders) if it had the power to accept or decline offers to join by members initially outside that bloc (here the third islander). But the situation might be that entry of an additional member (here the third island into a trading bloc with the first and second) would shift trade by the first from the second to the third island, in that way (further) benefiting the first, but leaving the second worse off relative to its previously trade related position. This is not a Pareto improving outcome of that change in bloc membership for the first two islands and, if Pareto improvement of that kind were a precondition for enlargement of a trading bloc, the second island would exercise a veto on that basis. If on the other hand, the second island had no veto and the first and third islands could form a new trading relationship by deposing the second, this would arguably lead to an inferior position for the second - and so a Pareto inferior outcome overall. [I use the word "arguably" here because a conclusion of Pareto inferiority depends here on the chosen comparator state. If the comparator state was the initial position then arguably trade between islands one and three would be Pareto preferred by all of the islanders since then islander two would be in their initial position and the other two islanders would be in position preferred by them.]

These illustrations make the general point that different overall outcomes may be obtained depending on whether or not members of existing trading blocs have a veto with reference to trade with economies initially outside such blocs e.g. on the basis that any augmentation of a trading bloc must be on the basis of Pareto improvement for (all of) its members. This idea is developed more formally via Theorems 1 through 3 below. It also suggests a point which is also developed further below, namely that in general the memberships of Pareto preferred trading blocs will not be independent of the sequence of their formation. It will be emphasized in Section 6 that in general the size of a bloc formed on the basis of successive applications of a Pareto improving criterion will depend, not just on the order in which members might join it, but on whether or not it is permissible to admit members more than one at a time. As preliminaries to these results some general theorems and associated Kemp-Wan-like results on customs unions and 
free trade areas are established in the next section.

\section{Gains From Exchange: Some General Results}

In this section free trade area and customs union results are developed in stages. First assume that concave functions $\theta_{r} f_{r}\left(y_{r i}, k_{r i}, l_{r i}, k_{i}^{r w}, l_{i}^{r w}\right), \theta_{s} f_{s}\left(y_{s i}, k_{s i}, l_{s i}, k_{i}^{s w}, l_{i}^{s w}\right)$ with $w=1,2$..n represent the preference relations of two blocs of nations $r=1, . . n_{1}$ and $s=n_{1+1} \ldots$ n over quantities $\mathrm{y}_{\mathrm{ri},} \mathrm{y}_{\mathrm{si}}$ of commodities $i=1,2 \ldots \mathrm{m}$ and of inputs of capital and of labour $k_{r i}, l_{r i}, k_{i}^{r w}, l_{i}^{r w}\left(\operatorname{resp} k_{s i}, l_{s i}, k_{i}^{s w}, l_{i}^{s w}\right)$ to production and to transportation services respectively. In that context assume that evaluations $\theta_{r} f_{r}\left(y_{r i}, k_{r i}, l_{r i}, k_{r}{ }^{r w}, l_{r}^{r w}\right)^{*}$, $\theta_{\mathrm{s}} \mathrm{f}_{\mathrm{s}}\left(\mathrm{y}_{\mathrm{si}}, \mathrm{k}_{\mathrm{si}}, 1_{\mathrm{si}}, \mathrm{k}_{\mathrm{r}}^{\mathrm{sw}}, 1_{\mathrm{r}}^{\mathrm{sw}}\right)^{*}$ are associated with an initial state and that $x_{i}{ }^{w w}\left(\right.$ resp $\left.x_{i}{ }^{w v}\right)$ are shipments of commodities i from nations $v=1,2 . . n$ to nations $\mathrm{w}=1,2 . . n$ (resp $w$ to $v$ ). Assume, too, that the available resources of labour and capital are $L_{r}{ }^{*}, L_{s}{ }^{*}$ and $K_{r}{ }^{*}, K_{s}^{*}$ and that outputs of type i and shipping services can be produced in nations $r, s$ via concave and differentiable production functions $g_{r}\left(k_{r i}, l_{r i}\right), g_{s}\left(k_{s i}, l_{s i}\right)$ and $g_{i}^{r w}\left(k_{i}^{r w}, l_{i}^{r w}\right) g_{i}^{s v}\left(k_{i}^{s w}, l_{i}^{s w}\right)$.

Under these assumptions consumption $\mathrm{y}_{\mathrm{ri}}$ of any commodity $\mathrm{i}$ in nation $\mathrm{r}$ is contingent on its availability $\mathrm{x}_{\mathrm{ri}}$ which will be constrained by the amount produced in nation $r, g_{r}\left(k_{r i} l_{r i}\right)$, plus imports $\Sigma x_{i}^{w r}$ less exports $\Sigma x_{i}^{r w}$. After including equivalent relations for nations $\mathrm{s}$ and production relations for shipped quantities $x_{i}^{r w}, x_{i}^{s w}$ and assuming that nations will seek to maximize their preferences given the initial state and that quantities $t_{i}{ }^{w w}, t_{i}{ }^{w v}$ correspond to tariffs placed on commodities i, consider the result in Theorem 1 below.

The result in Theorem 1 is very general. As a class of special cases with reference to a nation $\mathrm{r}$ in a bloc of nations $n=1,2 . . n_{1}$ and a nation $\mathrm{s}$ in a bloc of nations $s$ $=n_{1+1} . . n$ it states that in general a reduction of one or more of tariffs $t_{i}^{r s}, t_{i}^{s r}$ may lead to a Pareto preferred state for nations in both trading blocs. In the remainder of this section I consider specializations to yield Kemp-Wan-like cases in which the composition of the trading blocs is constant, before going on in the next section to consider further results stemming from changes in the membership of trading blocs simultaneously with changes in one or more tariffs.

\section{Theorem 1}

If $t_{i}^{v w}<t_{i}^{v w^{*}}$ and/or $t_{i}^{w v}<t_{i}^{w v^{*}}$ at least one i,r,s then:

$z_{1}^{*}=\operatorname{Max} \Sigma\left[\theta_{r} f_{r}\left(y_{r i}, k_{r i}, l_{r i}, k_{i}^{r w}, l_{i}^{r w}\right)+\Sigma\left[\theta_{s} f_{s}\left(y_{s i}, k_{s i}, l_{s i}, k_{i}^{s w}, l_{i}^{s w}\right)-\Sigma t_{i}^{v w^{*}} x_{i}^{v w}-\Sigma t_{i}^{w v^{*}} x_{i}^{w v}\right.\right.$

$$
\begin{array}{lr}
\text { st: } y_{r i} \leq x_{r i} & y_{s i} \leq x_{s i} \\
x_{r i} \leq g_{r i}\left(k_{r i}, l_{r i}\right)+\sum x_{i}^{w r}-\Sigma x_{i}^{r w} & x_{s i} \leq g_{s i}\left(k_{s i} l_{s i}\right)+\sum x_{i}^{w s}-\Sigma x_{i}^{s w}
\end{array}
$$




$$
\begin{array}{lc}
x_{i}^{r w} \leq g_{r i}{ }^{r w}\left(k_{r i}{ }^{r w}, l_{r i}{ }^{r w}\right) & x_{i}^{s w} \leq g_{s i}{ }^{s w}\left(k_{s i}^{s w}, l_{s i}^{s w}\right) \\
\Sigma k_{r i} \leq K_{r}{ }^{*} & \sum k_{s i} \leq K_{s}^{*}{ }^{*} \\
\Sigma l_{r i} \leq L_{r}{ }^{*} & \Sigma l_{s i} \leq L_{s}^{*} \\
\theta_{r} f_{r}\left(y_{r i}, k_{r i}, l_{r i}, k_{r}^{r w}, l_{r}^{r w}\right) \geq \theta_{r} f_{r}\left(y_{r i}, k_{r i} l_{j,}, k_{r}^{r w}, l_{r}^{r w}\right)^{*} \\
\theta_{s} f_{s}\left(y_{s i}, k_{s i}, l_{s i}, k_{s}^{s w}, l_{s}^{s w}\right) \geq \theta_{s} f_{s}\left(y_{s i}, k_{s i} l_{s i}, k_{s}^{s w}, l_{s}^{s w}\right)^{*}
\end{array}
$$

All variables nonnegative

$$
\begin{aligned}
& \geq \\
& z_{1}==\operatorname{Max} \Sigma\left[\theta_{r} f_{r}\left(y_{r i}, k_{r i} l_{r i}, k_{i}^{r w}, l_{i}^{r w}\right)+\Sigma\left[\theta_{s} f_{s}\left(y_{s i}, k_{s i}, l_{s i}, k_{i}^{s w}, l_{i}^{s w}\right)-\Sigma t_{i}^{v w} x_{i}^{v w}-\Sigma t_{i}^{v w} x_{i}^{w v}\right.\right. \\
& \text { st constraints of (I) }
\end{aligned}
$$

\section{Proof}

Any optimal solution to (I) is a feasible but not necessarily an optimal solution to (Ia).

Theorem 1 comprehends a number of special cases. First consider a class of prohibitively high tariff datum cases reminiscent of the initial no gift or exchange case in the motivating three island example of the previous section. Specifically: If $t_{i}^{v w^{*}}$ and $t_{i}^{w v^{*}}$ are sufficiently large all $\mathrm{i}, \mathrm{v}, \mathrm{w}$ then Theorem 1 potentially corresponds to cases in which no gifts or exchanges under an optimum to (I) are potentially Pareto inferior to gift, or barter, or trade related cases arising from sufficient reductions in one or more of tariffs $t_{i}^{v w^{*}}$ and/or $t_{i}^{w v^{*}}$ in (Ia).

This prohibitively high datum example suggests that it might sometimes be useful to consider the conditions underlying the last two constraints of (I) and thence of (Ia) as corresponding to an optimal solution to programme (I). That in turn suggests a potential for successive applications of Theorem 1 to prove the potential for successive Pareto improvements stemming from successive relaxations of tariffs, even given an invariant structure of trading blocs. (Using a different approach Kemp and Shimomura obtain an analogous result as a corollary of their extended Kemp-Wan proposition. See Kemp and Shimomura 2001.)

Since by assumption the relations $q_{r} f_{r}(), q_{s} f_{s}()$ and $g_{r i}\left(k_{r i}, l_{r i}\right), g_{s i}\left(k_{s i}, l_{s i}\right)$ and $g_{i}^{r w}\left(k_{r i}{ }^{r w}, l_{r i}{ }^{r w}\right), g_{r i}{ }^{s w}\left(k_{s i}{ }^{s w}, l_{s i}{ }^{s w}\right)$ are all concave and differentiable, it follows that (I) and (Ia) in Theorem 1 are concave programming problems and that the associated Kuhn-Tucker conditions associated with shipments $x_{i}^{v w}$ are sufficient for their solution. Among those conditions will be conditions which may be written in a stylized form as in (3.1). In (3.1) $p_{v i}, p_{w i}$ represent optimal values of the variables associated with the quantities $x_{v i}, x_{w i}$ via the third and fourth constraints of (I) and (Ia). With these interpretations (3.1) implies that at an optimum to (I) or (Ia), 
commodity $i$ will be shipped from region $\mathrm{v}$ to region $\mathrm{w}$, if at all, then only to the point where the price in region $\mathrm{w}$ is sufficient to recoup: i) its price in region $\mathrm{v}$ plus; ii) the shipping $\operatorname{cost} c_{i}{ }^{w w}$ from region $\mathrm{v}$ to $\mathrm{w}$ stemming from the resources $x_{i}^{v w}$ required for that shipment plus; iii) the associated unit tariff or subsidy, $t_{i}^{v w}$.

$$
p_{v i}+c_{i}{ }^{w w}+t_{i}^{v w} \geq p_{w i}
$$

Clearly, if $t_{i}^{v w}$ is sufficiently high in (3.1) (e.g. under the conditions of (I) of Theorem 1) then commodity i will not be shipped from nation $\mathrm{v}$ to nation $\mathrm{w}$ at all, whereas if $t_{i}^{v w}$ is then sufficiently reduced, commodity $i$ may optimally be shipped from nation $\mathrm{v}$ to nation $\mathrm{w}$. (This is another way of understanding the result stemming from one or more reductions $t_{i}^{v w}<t_{i}^{v w^{*}}$ in Theorem 1.)

As further examples of applications and developments of Theorem 1 it is instructive to consider results analogous to those deriving from Kemp and Wan (1986) with reference to customs unions. For those purposes, assume that (I) corresponds to an initial "tariff ridden" equilibrium and that the conditions of (Ia) are modified, first, so that in (Ia) the same tariff applies to commodities i for all nations shipping to nations $\mathrm{r}$ from nations $\mathrm{s}$. Secondly, assume that additional net import constraints $\Sigma x_{i}^{s r}-\Sigma x_{i}^{r s}=\Sigma x_{i}^{s r^{*}}-\sum x_{i}^{r s^{*}}$ for the bloc of nations $\mathrm{r}=1, . . n_{1}$ vis a vis the bloc of nations $s=n_{1+1} . . n$ are added to the constraints of (Ia) for each commodity $i$. These modifications lead to a correspondingly modified version of Theorem 1, viz:

\section{Theorem 2}

Denoting interbloc exchanges by the notation $\mathrm{x}_{\mathrm{i}}^{\mathrm{rs}}, \mathrm{x}_{\mathrm{i}}^{\mathrm{sr}}$ and intrabloc exchanges by the notations $\underline{x}_{i}^{r s}, \underline{x}_{i}^{s r}$ then for bloc $r=1,2, . . n_{1}$ there exist tariffs $\mathrm{t}_{\mathrm{i}}^{\mathrm{r}}$ such that $t_{i}^{r}=t_{i}^{s r}$ all s which imply:

$$
z_{2}^{*}=\operatorname{Max} \Sigma \theta_{r} f_{r}\left(y_{r i}, k_{r i}, l_{r i}, k_{i}^{r w}, l_{i}^{r w}\right)+\sum \theta_{s} f_{s}\left(y_{s i}, k_{s i}, l_{s i}, k_{i}^{s w}, l_{i}^{s w}\right)-\Sigma t_{i}^{v w^{*}} x_{i}^{v w}-\Sigma t_{i}^{v w^{*}} x_{i}^{v w}
$$

st constraints of (I) (II)

$$
\begin{aligned}
& \leq \\
& z_{2}==\operatorname{Max} \Sigma \theta_{r} f_{r}\left(y_{r i}, k_{r i}, l_{r i}, k_{i}^{r w}, l_{i}^{r w}\right)+\sum \theta_{s} f_{s}\left(y_{s i}, k_{s i}, l_{s i}, k_{i}^{s w}, l_{i}^{s w}\right)-\Sigma t_{i}{ }^{v w^{*}} x_{i}^{v w}-\sum t_{i}^{r} x_{i}^{s r}-\sum \underline{t}_{i}^{s r *} \underline{x}_{i}^{s r}
\end{aligned}
$$

st constraints of (I)

$$
\text { and } \Sigma x_{i}^{s r}-\Sigma x_{i}^{r s}=\Sigma x_{i}^{s *^{*}}-\Sigma x_{i}^{r r^{*}}
$$

where $x_{i}^{s r^{*}}, x_{i}^{r s^{*}}$ are associated with an optimal solution to (II).

\section{Proof}

A sufficient condition for this result is that $t_{i}^{r}<\min \left\{t_{i}^{s r^{*}}: r=1, . . n_{1}, s=s=n_{1+1} \ldots n\right\}$. Under that condition any optimal solution to (I) is a feasible but not necessarily an optimal solution to (Ia). 


\section{Corollary}

Associating Lagrange multipliers $\Delta_{i}^{s r}$ with the final net trade constraints in (IIa) inter bloc Kuhn-Tucker conditions analogous to (3.1) are generated from (IIa) respectively for imports $x_{i}^{s r}$ and for exports $\mathrm{x}_{\mathrm{i}}^{\mathrm{rs}}$ as follows:

$$
\begin{aligned}
& p_{s i}+c_{i}^{s r}+\Delta_{i}^{s r}+t_{i}^{s r} \geq p_{r i} \\
& p_{r i}+c_{i}^{r s}+\Delta_{i}^{s r}+t_{i}^{r s} \leq p_{s i}
\end{aligned}
$$

In context the quantities $\Delta_{i}^{s r}$ take on interpretations as exchange related transfer payments to consumers and suppliers of commodities $i$ in nations $r$.

In the introduction it was claimed that results in this paper can be seen as variants of results in Kemp and Wan (1986), Kemp (2000) and Kemp and Shimomura (2001). With reference to Kemp and Wan their key result refers to a customs union formed by a subset of nations. In that context Kemp and Shimomura summarize the properties of a CU and the Kemp Wan 1986 result as follows:

given any initial competitive but tariff ridden world equilibrium it is possible for any subset of two or more countries to form a customs union with a common tariff vector and with compensatory lump-sum intra union transfers such that in an assoc-iated world equilibrium no individual whatever his country of residence is made worse off and that, if a strict convexity condition on preferences is satisfied, all individuals within the customs union are better off. (Kemp, Shimomura 2001,p.64)

In more detail:

the defining feature of a customs union is a common tariff condition and a scheme of lump sum payments to households in the $\mathrm{CU}$ such that (a) the pre and post $\mathrm{CU}$ equilibrium aggregate net import vectors of the $\mathrm{CU}$ member countries are identical and (b) no household, whether inside or outside the $\mathrm{CU}$, is worse off after the foundation of the $\mathrm{CU}$.

Evidently with nations $\mathrm{r}=1, . . \mathrm{n}_{1}$ interpreted as members of a $\mathrm{CU}$ with common tariffs $\mathrm{t}_{\mathrm{i}}^{\mathrm{r}}$ Theorem 2 yields a similar conclusion to Kemp and Wan 1986 since in that case an optimum to (IIa) is consistent with conditions $\Sigma x_{i}^{s r}-\Sigma x_{i}^{r s}=\Sigma x_{i}^{s r^{*}}-\Sigma x_{i}^{r s^{*}}$ all i, with constraints $\theta_{r} f_{r}\left(y_{r i}, k_{r i}, l_{r i}, k_{r}^{r w}, l_{r}^{r w}\right) \geq \theta_{r} f_{r}\left(y_{r i}, k_{r i}, l_{j p}, k_{r}^{r w}, l_{r}^{r w}\right)^{*}, \theta_{s} f_{s}\left(y_{s i}, k_{s i}, l_{s i}, k_{s}^{s w}\right.$, $\left.l_{s}^{s w}\right) \geq \theta_{s} f_{s}\left(y_{s i}, k_{s i}, l_{s i}, k_{s}^{s w}, l_{s}^{s w}\right) *$ all r,s and with compensatory payments $\Sigma \Delta_{i}^{s r}\left(x_{i}^{s r}-x_{i}^{r s}\right)$ to nations $\mathrm{r}$.

Three other variants of the result in Theorem 2 are also readily obtainable. These are the subject of Section 4. 


\section{Three variants of Theorem 2 and their Implications}

For the first variant of Theorem 2 add one or more nation-specific conditions of the form $x_{i}^{s r}=x_{i}^{s r *}$ to (IIa) in Theorem 2. If $\underline{\Delta}_{i}^{s r}$ are the dual variables associated with these additional constraints then the analogue of condition (3.2) in Section 3 is:

$$
p_{s i}+c_{i}^{s r}+\underline{\Delta}_{i}^{s r}+\Delta_{i}^{s r}+t_{i}^{s r} \geq p_{r i}
$$

Interpreting $\underline{\Delta}_{i}^{s r}$ as marginal changes in the tariff vectors $t_{i}^{s r}$ consequent upon the addition of constraints $x_{i}^{r s}=x_{i}^{r s^{*}}$, these variations and their implications appear to be analogous to those obtained in Kemp, (2000), viz:

Let us now disturb the Kemp-Wan CU by marginally manipulating the tariff vector of at least one member country so that the aggregate net import vector of member countries is unchanged. The new trading association obtained in this way is, like the Kemp-Wan CU itself, an FTA (Frade Trade Area). However, it is not possible to ensure that all member households are better off than in the Kemp-Wan CU. (Kemp (2000) p2.)

The analogue of the conclusion in the final sentence of this quote follows here from the fact that, with the addition of constraints of the form $x_{i}^{s r}=x_{i}^{s r *}$, the consequent problem is more tightly constrained than (IIa) and therefore cannot yield a higher optimum than (IIa). [By considering the "tariff ridden" case with $t_{i}^{w w^{*}}, t_{i}^{w v^{*}}$ all i,v,w first, followed by an FTA-like case with one or more additional constraints $x_{i}^{s r}=x_{i}^{s r^{*}}$ with associated conditions $t_{i}^{s r}<t_{i}^{s r^{*}}$ followed by the less restrictive common tariffs $t_{i}^{r}<\min \left\{t_{i}^{s r^{*}}: r=1, . . n_{1}, s=s=n_{1+1} \ldots n\right\}$ case, the reader can easily obtain a result analogous to the main "generalized proposition" result on $\mathrm{p} 2$ of Kemp 2000, to the effect that such a CU is Pareto preferable to such an FTA, which in turn is Pareto preferable to such a tariff ridden initial state.]

A second class of variants of Theorem 2 would use arguments similar to those which have been used to obtain various kinds of customs union result for nations $r=1, . . n_{1}$, to obtain complementary results for nations $s=n_{1+1} . . n$ via sufficient tariff reductions $t_{i}^{s}<\min \left\{t_{i}^{r s^{*}}: s=n_{1+1} . . n\right\}$ in Theorem 2. These results might follow in three distinct ways, either immediately via the analogue of Theorem 2 for nations $s=n_{1+1} . . \mathrm{n}$ starting from a customs union chosen, for example via successive applications of Theorem 2, by nations $r=1, . . n_{1}$, or by successive relaxations of tariffs by members of the trading bloc $s=n_{1+1} . . n$ in a manner analogous to that considered in Kemp and Shimomura 2001, or in stages, but now for nations $s=n_{1+1}$ ..n, from a tariff ridden economy to an FTA to a customs union in a manner similar 
to the "generalized proposition of Kemp 2000, which was cited in connection with the first variant above of Theorem 2 in this Section.)

A third class of variants of Theorem 2 is one not considered by Kemp, Wan or Shimomura, or others, namely mobile factor variants of the previous results. Specifically: denoting international transfers of labour and of capital by $l_{i}^{w w}, l_{i}^{w v}$ and $k_{i}{ }^{v w}, k_{i}{ }^{w v}$ respectively and associating correspondingly tariff-like penalties or subsidies $d_{i}{ }^{v w^{*}}, d_{i}{ }^{w v^{*}}, e_{i}{ }^{v w^{*}}, e_{i}{ }^{w{ }^{*}}\left(\operatorname{resp} d_{i}{ }^{v w}, d_{i}{ }^{w v}, e_{i}{ }^{v w}, e_{i}{ }^{w v}\right)$ with those quantities, an extended form of Theorem 2 applies via the systems (III) and (IIIa) and Theorem 3 below.

\section{Theorem 3}

If at least one of the conditions $d_{i}^{v w}<d_{i}^{v w^{*}}, d_{i}^{w v}<d_{i}^{w v^{*}}, e_{i}^{v w}<e_{i}^{v w^{*}}, e_{i}^{w v}<e_{i}^{w v^{*}}$ obtains, the following result holds even if $t_{i}^{v w}=t_{i}^{v w^{*}}$ and $t_{i}^{w v}=t_{i}^{w v^{*}}$ all i,r,s:

$$
\begin{aligned}
& z_{3}{ }^{*}=\operatorname{Max} \sum \theta_{r} f_{r}\left(y_{r i}, k_{r i}, l_{r i}, k_{r i}{ }^{r w}, l_{r i}{ }^{r w}, k_{r}^{r w}, l_{r}^{r w}\right)+\sum \theta_{s} f_{s}\left(y_{s i}, k_{s i}, l_{s i}, k_{s i}{ }^{s w}, l_{s i}^{s w}, k_{s}^{s w}, l_{s}^{s w}\right) \\
& -\Sigma t_{i}^{v w^{*}} x_{i}^{v w}-\Sigma t_{i}^{w v^{*}} x_{i}^{w v}-\Sigma d_{i}^{v w^{*}} k_{i}^{v w}-\Sigma d_{i}^{w v^{*}} k_{i}^{w v}-\Sigma e_{i}^{v w^{*}} l_{i}^{v w}-\Sigma e_{i}^{w v^{*}} l_{i}^{w v} \\
& \text { st: } \quad y_{r i} \leq x_{r i} \\
& x_{r i} \leq g_{r i}\left(k_{r i}, l_{r i}\right)+\sum x_{i}^{w r}-\sum x_{i}^{r w} \quad x_{s i} \leq g_{s i}\left(k_{s i}, l_{s i}\right)+\sum x_{i}^{w s}-\sum x_{i}^{s w} \\
& x_{i}^{r w} \leq g_{r i}{ }^{r w}\left(k_{r i}{ }^{r w}, l_{r i}{ }^{r w}\right) \quad x_{i}^{s w} \leq g_{r i}{ }^{s w}\left(k_{s i}{ }^{s w}, l_{s i}{ }^{s w}\right) \\
& \Sigma k_{r i}+\sum k_{r i}{ }^{r w} \leq K_{r}{ }^{*}+\sum k_{r}{ }^{w r}-\Sigma k_{r}^{r w} \quad \Sigma k_{s i}+\sum k_{s i}{ }^{r w} \leq K_{s}{ }^{*}+\sum k_{s}{ }^{w s}-\Sigma k_{s}^{s w} \\
& \Sigma l_{r i}+\Sigma l_{r i}{ }^{r w} \leq L_{r}{ }^{*}+\Sigma l_{r}^{w r}-\Sigma l_{j}^{r w} \quad \Sigma l_{s i}+\Sigma l_{s i}{ }^{r w} \leq L_{s}{ }^{*}+\Sigma l_{s}^{w s}-\Sigma l_{s}^{s w}
\end{aligned}
$$

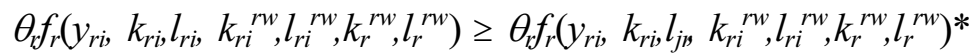

$$
\begin{aligned}
& \theta_{s} f_{s}\left(y_{s i}, k_{s i}, l_{s i}, k_{s i}^{s w}, l_{s i}^{s w}, k_{s}^{s w}, l_{s}^{s w}\right) \geq \theta_{s} f_{s}\left(y_{s i}, k_{s i}, l_{s i}, k_{s i}^{s w}, l_{s i}^{s w}, k_{s}^{s w}, l_{s}^{s w}\right)^{*}
\end{aligned}
$$

All variables nonnegative

$$
\begin{aligned}
& \leq \\
& z_{3}=\operatorname{Max} \Sigma \theta_{r} f_{r}\left(. y_{r i}, k_{r i}, l_{r i}, k_{i}^{r w}, l_{r i}^{r w}, k_{r}^{r w}, l_{r}^{r w}\right)+\sum \theta_{s} f_{s}\left(y_{s i}, k_{s i}, l_{s i}, k_{s i}{ }^{s w}, l_{s i}^{s w}, k_{s}^{s w}, l_{s}^{s w}\right) \\
& -\Sigma t_{i}^{v w} x_{i}^{v w}-\Sigma t_{i}^{w v} x_{i}^{w v}-\Sigma d_{i}^{v w} k_{i}^{v w}-\Sigma d_{i}^{w v} k_{i}^{w v}-\Sigma e_{i}^{v w} l_{i}^{v w}-\Sigma e_{i}^{w v} l_{i}^{w v}
\end{aligned}
$$$$
\text { st constraints of (III) (IIIa) }
$$$$
\text { and } \Sigma x_{i}^{s r}-\Sigma x_{i}^{r s}=\Sigma x_{i}^{s r}-\Sigma x_{i}^{r s^{*}}
$$

where

$x_{i}^{s r *}, x_{i}^{r s^{*}}$ are associated with an optimal solution to (III).

\section{Proof}

Any optimal solution to (III) is a feasible but not necessarily an optimal solution to (IIIa)

The specifications (III) and (IIIa) in Theorem 3 extend the specifications (II), (IIa) in Theorem 2 and associated results to admit mobility of labour and of capital between nations, in that way generating the potential for Pareto improving 
outcomes for sufficiently low values of the corresponding quantities $d_{i}^{v w}, d_{i}^{w v}, e_{i}^{v w}$, $e_{i}^{w v}$. Conversely, conditions prohibiting such mobility would in general lead to relatively Pareto inferior states, since a relatively more constrained system cannot yield a higher optimum. [In this context it may be helpful to use the relatively less constrained example of (IIa), which implicitly prohibits movement of factors, vis a vis (IIIa), which will generally permit such movement, albeit usually at a correspondingly nonzero tariff-like expense $d_{i}^{v w}, d_{i}^{w v}, e_{i}^{v w}, e_{i}^{w v}$.]

Clearly variants of Theorem 3 could be used to extend all of the results which have been developed via Theorem 2 to the more general class of mobile factor cases comprehended by Theorem 3. (In that context, too, the systems (III) and (IIIa) could easily be extended to endogenize resources which may be associated with facilitating mobility of factors between nations in a manner similar to that which has been used to endogenize resources required for the transport of produced commodities.)

More positively, the mobile factor cases covered by Theorem 3 may seem more consistent in principle with empirical circumstances such as the EC related labour and capital mobility example which was mentioned in the Introduction than nonmobile factor cases, including those of Kemp and Wan (1986), Kemp (2000) and Kemp and Shimomura (2001).

\section{Non Pareto Improving Conditions}

Developments in the previous two sections have focussed on variants and extensions of results on customs unions and free trade areas analogous to those obtained by Kemp and Wan and Shimomura. As expressed here (supported by appropriate quotes from those authors) those results all share a common feature, namely that a tariff reduction will never deliver a Pareto inferior outcome - even for members outside a customs union or free trade area. [Parenthetically I have implicitly taken a sharply different interpretation of Kemp and Wan (1986) from others. In particular Winters 1997 asserts that "Kemp and Wan did not deal with changes in non-member welfare." For more on that point see Winters' paper noting of course that it antedates Kemp 2000 and Kemp and Shimomura 2001 whom I have interpreted here as talking a contrary view of the original Kemp-Wan work.]

In any case the essentially Pareto improving character of the results which have been developed in Sections 3 and 4 differ sharply from the motivating three island 
example which I used in Section 2. In that example Nation 1 joined Nation 2 to gain a Pareto improved state. It was then suggested that Nation 3 might join Nation 1 in such a way as in effect to depose Nation 2 and that, relative to the previously positive level of exchange between Nations 1 and 2, this would be a less preferred outcome for Nation 2 and in that sense would be associated with a Pareto inferior outcome for all three nations relative to a previously positive level of trade between nations 1 and 2. Why this difference between implications of the example in Section 2 and implications of the Kemp-Wan-Shimomura-like analyses and extensions in Sections 3 and 4?

The fundamental reason for this difference is that specifications in Sections 3 and 4 all explicitly prohibit a Pareto inferior outcome for any nation - be it inside or outside a customs union or free trade area. This explicit prohibition seems to be a very strong condition and indeed an empirically implausible one. Why should the members of a customs union or free trade area seek a a more preferable state for members outside their union than would otherwise be attainable - especially if that may have the consequence of a less preferred outcome for one or more members of their customs union or free trade area? One response might be pragmatic and to the effect that, if the memberships of blocs are invariant, then cutting tariffs in such a mutually beneficial way might suggest a virtuous circle according to which each bloc might agree to successive tariff cuts benefiting both themselves and others. But another kind of response might be much less friendly and to the effect that in practice trading blocs have opportunities to "cherry pick" partners from each other.

Another and complementary kind of empirical implausibility of analyses in Sections 3 and 4 is the explicit assumption that, if outcomes of the associated optimizations are to obtain, then the consequent optima and, more specifically, the consequent levels of net imports (e.g. via the final constraints of (IIa) or (IIIa)) must be acceptable to all parties regardless of the nation or bloc of nations which reduces tariffs. Such an assumption seems simply implausible. By contrast in practice the preferences of one or more nations may effectively be given no weight in the relevant optimization or constraints. In such a case the outcome of a tariff reduction by existing members of a customs union vis a vis a complementary bloc of nations and/or a tariff reduction by an enlarged customs union vis a vis a complementary bloc of nations, may yield a less preferred outcome for one or more members of that complementary bloc, e.g. by reduction or removal of trade relations with them.

A motivating example here is the selection by the EC of additional members 
from the East European bloc of nations for membership of an enlarged EC customs union and free trade area. In that case it is not at all clear that the outcome of such a change in memberships has been - or indeed is intended to be - Pareto improving in the sense of leading to a preferred state for all nations concerned be they in the EC, e.g. Poland or Hungary or not in the EC, e.g. Ukraine or Belarus. Mathematically, cases in which accessions of members $s=n_{1+1} \ldots$ were being considered one or more at a time would be similar to those considered under Theorem 3, except that the index s in the relations $\Sigma \theta_{s} f_{s}\left(. y_{s i}, k_{s i}, l_{s i}, k_{i}^{s w}, l_{i}^{s w}, k_{s}^{s w}, l_{s}^{s w}\right)$ in the maximands of (III) and (IIIa) would refer only to nations s potentially augmenting the initial bloc of nations $r=1,2 \ldots n_{1}$ with the indices of the net import related constraints in (IIIa) modified accordingly. In that way the maximands of (III) and (IIIa) would relate only to an existing bloc and potentially acceding nations or groups of nations and, in effect, the preferences of the nations remaining in the complementary bloc would have no weight in the objectives of (III),(IIIa).

Another way of appreciating the point in the previous paragraph is by means of the transfer payments stemming not only explicitly, via the quantities $\Delta_{i}^{s r}, \Delta_{i}^{s r}$, from additional net import related constraints in (IIIa), but also stemming implicitly from the welfare Pareto improving conditions of Theorem 3. Each kind of transfer will have the effect of redistributing resources between existing members of a customs union. Not only that but under the conditions of (IIIa) and Theorem 3 such redistributions will also take place to sustain or exceed given levels of preferences for nations outside that customs union, in the face of changes in tariffs. In practice, that may imply an effective subsidy to one or more nations, whether inside or outside a trading bloc, in such a way as to sustain otherwise unsustainable levels of net imports. The three island example is again helpful here. First, consider tariffs within a trading bloc: If the three islands are members $r=1,2,3$ of a bloc $r=1,2, . . n_{1}$ and if Island 3 has an absolute advantage over Island 2 and if tariffs are reduced in the order Islands 1 and 2 followed by Island 3 in a manner consistent with earlier customs union related developments here, then the Pareto improving requirement in those developments would in effect require transfers to island 3 within the trading bloc $r=1,2, . . n_{1}$ to compensate for the loss of trade with island 1. Next, consider an interpretation between trading blocs according to which nations 1 and 3 in the island example are in a first trading bloc and nation 2 is in a second. Then lower tariffs between 1 and 3 while maintaining zero trade with 2 . Again nation 3 would in effect obtain persistent subsidies not only implicitly from the Pareto improving criterion for nations in the first trading bloc, but also explicitly via the 
quantities $\underline{\Delta}_{i}^{s r}, \Delta_{i}^{s r}$ associated with the (zero) net import restrictions.

More subtly, even where the Kemp-Wan-Shimomura approach (as well as extensions of it here) focuses on opportunities for attaining unanimously (Pareto) preferred states via successive reductions in tariffs, e.g. for certain commodities $i$ by members of a trading bloc $r=1,2, . . n_{1}$, followed by reductions in tariffs for those of other commodities by members of a complementary trading bloc $s=n_{1+1} \ldots . n$, those analyses do not consider the fact that, in general, gains to members of tariff reduction induced trading blocs will be path dependent. The existence of path dependent outcomes in turn will lead to incentives for nations to act strategically vis a vis particular stages in that sequence. The island example has illustrated this idea throughout the paper since it is clear from that example that outcomes for all three nations would be very different, if in one case Islands 1 and 2 formed a bloc which was then in effect enlarged to include Island 3, and in another case Islands 1 and 3 formed a bloc which was then potentially enlarged to include Island 2. For the island example, under the assumptions of Theorem 3 Island 2 would be left outside the union of Islands 1 and 3 in the latter case, but in the former case would be required to be retained inside a potentially enlarged customs union made up of Islands 1,2 and 3 .

To underline this path dependence point I consider a different approach in the next Section which links these developments to ideas from game theory.

\section{Sequences of Formation of Coalitions and Some Game Theoretic Ideas}

It was noted in the context of Theorems 2 and 3 and developments in Sections 3 and 4 that Pareto improving sequences of tariff reductions could be constructed corresponding in effect to nations joining a customs union one at a time. It was noted there, too, that that result is analogous to the result in Kemp and Shimomura (2001). In each of those cases the Pareto improving result would be obtained, if necessary, by means of appropriate transfers in effect to compensate all nations whether or not those nations were in the customs union since, under the assumptions of those results, all nations would be at least Pareto indifferent between the initial and the final states in response to each tariff reduction. But that raises the question why compensate nations outside a free trade area or customs union? This question was addressed in Section 5 by relaxing the requirement that nations $s=n_{1+1} \ldots n$ outside a bloc of nations $r=1,2 \ldots n_{1}$ be at least as well off as they 
were prior to any internal tariff reductions by members of the bloc $r=1,2 \ldots n_{1}$. In Section 5, too, a second assumption of the analyses in Sections 3 and 4 (and also in the work of Kemp and Wan and Shimomura) was also relaxed, namely that the membership of trading blocs be invariant as tariffs are changed.

Section 5 concluded by using the three island example to illustrate the more general point that incremental gains to nations joining a customs union, as measured by their preferences, will generally depend on the sequence with which they join it. The purpose of this sixth Section is to underline these ideas in a different way by contrasting developments in this paper - which focus on individual and consequently non transferable utility based measures of gain - with ideas and interpretations relating to Shapley's own constructive interpretation of the Shapley Value in transferable utility characteristic function games.

In his seminal paper Shapley (1953) motivates his value for N player characteristic function games as follows:

The players...agree to play the game $\mathrm{v}$ in a grand coalition formed in the following way: 1). Starting with a single member the coalition adds one player at a time until everyone has been admitted. 2). The order in which the players are to join is determined by chance, with all arrangements equally probable. 3). Each player on his admission, demands and is promised an amount which his adherence contributes to the value of the coalition (as determined by the function $\mathrm{v})$.The grand coalition then plays the game "efficiently" so as to obtain the amount v(N) -exactly enough to meet all promises. (Shapley, op cit p.78.)

I have chosen to consider this interpretation of the Shapley value here because it seems to me that in analyses in earlier sections trading blocs have the character of coalitions and growth of FTAs, either within trading blocs, or by including members of complementary trading blocs via successive applications respectively of Theorem 1 or of the variant of Theorem 3 considered in Section 5, might seem evocative of the process considered by Shapley under 1) in the above quotation. More deeply, surely the bargaining associated with decisions concerning whether or not to change a tariff or to increase the membership of a trading bloc are fundamentally strategic in character. From that perspective assumptions familiar in the context of the Shapley value can provide a useful datum for the purpose of comparison and contrast with analyses and results here.

First and foremost the Shapley value stemming from the process in the above quotation relates to transferable utility - players share out the value $\mathrm{v}(\mathrm{N})$ in fractions stemming from rules 2) and 3 ) in the above process. By contrast 
developments here relate to non-transferable utility cases in which gains (or losses) to players $r, s$ in blocs $r=1, \ldots$ stemming from the enlargement of free trade areas or of customs unions are measured according to those players' own preference relations. [While this statement is strictly correct the reader might note that constraints in (III) and (IIIa) and Theorem 3 requiring Pareto improvement and constancy of net external trade nevertheless indirectly transfer utility between nations in response to changes in tariffs, e.g. via consequential transfers $\Delta_{i}^{s r}, \Delta_{i}^{s r}$ in conditions (4.1).]

Secondly, the magnitude of utility based payoffs to nations here will in general depend not only on the membership of a grand coalition if it forms (e.g. a coalition of islands 1,2,3), but on the sequence of formation of that grand coalition if it forms. By contrast, in the context of the Shapley value as in all standard representations of characteristic function games, payoffs to coalitions depend only on the membership of those coalitions and not the sequences for processes of their formation. For example in the case of the Shapley value the value $\mathrm{v}(\mathrm{N})$ imputed to the grand coalition of $\mathrm{N}$ players $\mathrm{N}$ in an $\mathrm{N}$ person game is independent, by assumption, of the process of formation of that coalition.

Third, if accessions are to be Pareto improving at each stage for members eventually included in a trading bloc it may be preferable for nations to join more than one at a time. An extension of the islands example will serve not only to illustrate this point but to motivate the more general point that, if it is desired to enlarge a customs union in a manner which will at each stage by Pareto enhancing both for its existing members and for members to be included in the union at subsequent stages, then in general it will be necessary to consider the admission of members more than one at a time. Accordingly consider initial conditions in which four nations each subsist in isolation. Then, by Theorem 2, in general exchange between any pair or collection of these nations will be at least weakly Pareto preferred to a condition of no exchange. With a view to the consideration of applications to EC enlargement consider a sequence in which these four nations first form two pairs $\{1,2\}$ and $\{3,4\}$ (see Figures 1 and 2 ) and then nation 3 is induced to leave the second pair and join 1 and 2 (e.g. by the variable bloc size variant of Theorem 3 considered in Section 5) so that the outcome of that sequence is the partition $\{1,2,3\}$ and $\{4\}$, as illustrated schematically in Figure 3 (Arrows indicate net exports.): 
Figure 1.

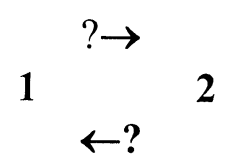

Figure 2.

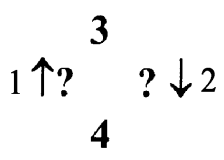

Figure 3.

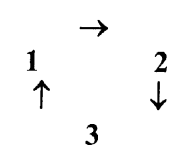

4

If the formation of coalitions of nations $\{1,2\}$ and $\{3,4\}$ were undertaken in accordance with the conditions of Theorem 3 then $\{1,2\}$ (resp $\{3,4\}$ ) would at least weakly Pareto dominate $\{1\},\{2\}$ (resp $\{3\},\{4\})$. Similarly if the formation of the coalition $\{1,2,3\}$ as a combination of $\{1,2\}$ and $\{3\}$ where nation 3 defected from the coalition $\{3,4\}$ was undertaken in accordance with the variable bloc variant of Theorem 2 considered in connection with Theorem 3, then, for nations 1,2,3 the coalition $\{1,2,3\}$ would at least weakly Pareto dominate $\{1,2\}$ and $\{3,4\}$. Of course, however, in general in such circumstances nation 4 would reach a Pareto inferior state as a consequence of defection of nation 3 from the coalition $\{3,4\}$. (Exceptions would only occur if for nation 4 the states $\{4\}$ and $\{3,4\}$ were indifferent to each other.) Further, if nations agreed to admit other nations only if that would lead to at least weakly Pareto dominating states, there may be cases where nations $1,2,3$, having formed a trading bloc $\{1,2,3\}$ would not choose to admit nation 4 to form a grand coalition as a combination of $\{1,2,3\}$ with $\{4\}$.

Now consider an alternative scenario in which nations 1,2 and 3,4 formed coalitions $\{1,2\}$ and $\{3,4\}$ as above but now, rather than admitting 3 and then deciding whether or not subsequently to admit 4 , nations 1,2 decide to offer admission simultaneously to 3 and 4 - that is to seek a grand coalition in just one further step. By corresponding applications of Theorem 2 the formation in this way of the grand coalition $\{1,2,3,4\}$ would be at least weakly Pareto preferable to continuation of the partitioned trading blocs $\{1,2\}$ and $\{3,4\}$. In this case, in distinction from that in the previous paragraph where a grand coalition $\{1,2,3,4\}$ would form, if at all, then only following a prior partition $\{1,2,3\},\{4\}$, there would be no possibility of Pareto inferior outcomes for $\{4\}$ as a consequence of the prior formation of a coalition $\{1,2,3\}$ or for any of the nations $1,2,3$ as a consequence of the admission of nation 4 .

This example illustrates and motivates the more general point that, if a customs union has the objective of processes of enlargement which will Pareto enhancing at each stage for members of any such enlarged union, then it may be that processes of enlargement by means of blocs will be in that sense Pareto preferable to 
processes of enlargement one nation at a time. From the perspective of policy this observation provides one kind of rationale for the fact that the EC has not been enlarged one nation at a time.

\section{Conclusion}

The main purpose of this paper has been to show how extremal characterizations of potentially Pareto improving conditions for exchange by augmentation of trading blocs can be useful in considering structures for markets with potentially increasing numbers of traders in one trading bloc vis a vis its complement. The paper has been motivated in part by considering potential applications to the identification of mutually advantageous processes of EC enlargement vis a vis potentially disadvantageous trade related consequences for non EC nations. In those contexts two key conclusions have been established-first that there may be advantage in the admission of more than one nation at a time since admission of nations one at a time is not necessarily Pareto enhancing at each stage for members of the ultimately formed customs union. Secondly, using arguments analogous to those used by Kemp and Wan and others it has been shown that there may be cases where the admission of nations to a customs union or free trade area while at the same time allowing taxes or subsidies $\underline{\Delta}_{i}^{\text {sr }}+\Delta_{i}^{\text {sr }}$ to exchanged commodities may be Pareto superior to an alternative regime in which no commodity related taxes or subsidies would be permitted. It follows that transactions between nations within trading blocs based on principles of absence of subsidies to exchange or trade may be Pareto disimproving vis a vis those nations and by extension - for regional blocs of nations.

I close by suggesting two kinds of generalization. First: while emphasis has principally been on gains potentially accruing to members of trading blocs from increasing opportunities to exchange or trade commodities i between them, extensions of Theorems 1,2 via Theorem 3 also admit further extensions and interpretations that correspond to gains potentially stemming from migration of factors of production. Secondly; Theorems 1,2,3 and associated developments extend in principle to any finite number of nations. Nevertheless, for purposes of explanation attention has been focussed here on examples with four or less nations. If larger numbers of nations are explicitly considered, correspondingly richer kinds of predictions will emerge - especially with reference to implications for intra bloc trading patterns. Figures $4 \mathrm{a}$ and $4 \mathrm{~b}$ illustrate just one possibility in which there are 
Figure 4a

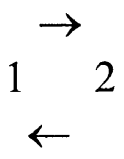

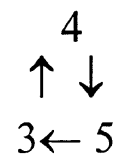

Figure 4b

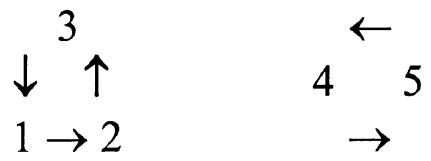

five nations in two blocs the first bloc with two way trade between nations 1,2 and the second with a triangle of trade between nations 3,4,5. (Arrows indicate net exports.):

If nation 3 moves from the second to the first bloc in a manner consistent with the variable bloc extension of Theorem 3, so leading to a Pareto improvement for members of the first bloc plus nation 3 , then the pattern of intra bloc exchanges might become as in Figure 4b. Clearly the pattern and the volume of exchange has radically changed for the majority of these trading nations - and not just for nation 3 . If the five nations exported five distinct commodities not only would volumes and directions of exports change radically: so would their content.

Received 11 February 2004, Accepted 12 August 2004

\section{References}

Kemp, M.C., 2000, "Welfare Comparisons of Customs Unions and other Free Trade Associations", Pacific Economic Review, 5,1,1-3.

Kemp, M.C. and K.Shimomura, 2001, "A Second Elementary Proposition Concerning the Formation of Customs Unions", Japanese Economic Review, 52,1,64-69.

Kemp, M.C. and H.Y.Wan, 1986. "An Elementary Proposition Concerning the Formation of Customs Unions", Journal of International Economics, 6, 95-97.

Shapley, L.S., 1953, A Value for N Person Games, Contributions to the Theory of Games II, pp 307-317. Also ch 7 in H.Kuhn, ed, Classics in Game Theory, Princeton University Press, 1997.

Winters, A., 1997, "Regionalism and the Rest of the World: The Irrelevance of the KempWan Theorem", Oxford Economic Papers, 49, 228-234. 